\author{
MITSUBISHI ELECTRIC RESEARCH LABORATORIES \\ http://www.merl.com
}

\title{
Wireless Power Transmission Efficiency Enhancement With Metamaterials
}

\author{
Bingnan Wang, Tamotsu Nishino, Koon Hoo Teo
}

TR2010-078 September 2010

\begin{abstract}
In this paper, a wireless power transfer system with magnetically coupled resonators is studied. The idea to use metamaterials to enhance the coupling coefficient and the transfer efficiency is proposed and analyzed. With numerical calculations of a system with and without metamaterials we show that the transfer efficiency can be improved with metamaterials.
\end{abstract}

ICWITS

This work may not be copied or reproduced in whole or in part for any commercial purpose. Permission to copy in whole or in part without payment of fee is granted for nonprofit educational and research purposes provided that all such whole or partial copies include the following: a notice that such copying is by permission of Mitsubishi Electric Research Laboratories, Inc.; an acknowledgment of the authors and individual contributions to the work; and all applicable portions of the copyright notice. Copying, reproduction, or republishing for any other purpose shall require a license with payment of fee to Mitsubishi Electric Research Laboratories, Inc. All rights reserved.

Copyright (C) Mitsubishi Electric Research Laboratories, Inc., 2010

201 Broadway, Cambridge, Massachusetts 02139 



\title{
Wireless power transmission efficiency enhancement with metamaterials
}

\author{
Bingnan Wang ${ }^{(1)}$, Tamotsu Nishino ${ }^{(2)}$, Koon Hoo Teo*(1) \\ (1) Mitsubishi Electric Research Laboratories, 201 Broadway Ste 8, Cambridge, MA 02139 USA \\ (2) Mitsubishi Electric Corporation Information Technology R\&D Center, 5-1-1 Ofuna, \\ Kamakura, Kanagawa 247-8501, Japan \\ E-mail: teo@merl.com
}

\begin{abstract}
In this paper, a wireless power transfer system with magnetically coupled resonators is studied. The idea to use metamaterials to enhance the coupling coefficient and the transfer efficiency is proposed and analyzed. With numerical calculations of a system with and without metamaterials, we show that the transfer efficiency can be improved with metamaterials.
\end{abstract}

\section{Introduction}

In recent years, more efforts have been put into the research and development on wireless power transfer technologies, aiming to cut the power cord for various electrical and electronic devices. Indeed, the applications of wireless power transfer technologies can be very broad, from lowpower consumer electronics and implanted medical devices to high-power industrial and electric vehicle applications. Wireless power transfer has been pursued by Tesla in the beginning of $20^{\text {th }}$ century. However, safety is a main concern to prevent it from applications in everyday life. Later research work has been focused on technologies with magnetic coupling, as they are less sensitive to surroundings and safer for humans.

With magnetic coupling or inductive coupling, very high efficiency between transmitter and receiver coils can be achieved at distances less than a few centimeters [1]. In other words, the transmitter and receiver must be strongly coupled, with coupling coefficient $k=M / \sqrt{L_{1} L_{2}}$ greater than 0.9 , where $L_{1}$ and $L_{2}$ are the inductance of transmitting and receiving coils and $M$ is their mutual inductance. When the transmitter and receiver are working at resonant frequency, the range can be extended significantly [2][3][4][5][6]. With a much smaller coupling coefficient (usually less than 0.1 ), a relatively high efficiency can still be achieved. The key for magnetic resonant coupling is to keep the coupling rate of transmitter and receiver larger than the loss rate, or to keep $k^{2} Q_{1} Q_{2}$ as large as possible, where $Q_{1}$ and $Q_{2}$ are the quality factor of the resonance of transmitter and receiver [4][5]. The principle has been known for decades; however, the research was limited to wirelessly powering implanted medical devices [2][3][4], where the required distance is just a few centimeters and the required power level is low. Recently, wireless power transfer in mid-range (a few centimeters to 2 meters) with high efficiency and relatively high power level (on the order of 100W) is realized [5][6]. Again, the key is to design transmitter and receiver with high $Q$ resonators and maintain the coupling coefficient so that $k^{2} Q_{1} Q_{2}$ is much larger than one. 
It is known that the coupling coefficient between two coaxial coils is proportional to the cubic of the distance between coils. So, at a certain distance, when the $\mathrm{Q}$ factors are fixed, we have to improve the coupling coefficient in order to improve the overall transfer efficiency. In this paper, we propose the idea to use negative-index metamaterials to enhance the coupling coefficient between transmitter and receiver, and improve the transfer efficiency.

\section{Negative-index metamaterials and evanescent wave amplification}

Metamaterials are usually periodically arranged artificial structures that show peculiar properties such as negative refraction (see, for example, [7], and references therein). The elemental structures of the metamaterials are typically much smaller in size relative to the wavelength and electromagnetic (EM) properties of metamaterials are obtained from these elemental structures instead of the composite materials. Macroscopic parameters such as electric permittivity $\varepsilon$ and magnetic permeability $\mu$ can then be used to describe the EM properties of the metamaterials [6]. Almost arbitrary parameters can be achieved by carefully designing the elemental structures of metamaterials. Negative-index metamaterials (NIMs) are the first and one of most important metamaterials. It was first proposed by Veselago theoretically in 1968 [8], further investigated with realistic designs by Pendry in 2000 [9], and experimentally realized in 2001 [9]. Pendry showed that EM waves can propagate in a medium with $\varepsilon$ and $\mu$ both negative. Negative refraction will happen at the interface between a regular medium ( $\varepsilon$ and $\mu$ both positive) and such a metamaterial [9]. With a flat slab of NIM, negative refraction happens at both interfaces. So the propagating waves of an object can be focused inside the NIM slab and refocused outside. Besides, Pendry also showed that evanescent waves can be amplified inside a NIM slab. With a NIM slab, evanescent waves of an object can be restored too. So a NIM slab recovers both propagating waves and evanescent waves of an object and makes a "perfect lens". For a NIM slab with thickness t, the image will be $2 t$ away from the object. In other words, the effect of the lens is to translate the object by a distance $2 t$.

The properties of NIMs, especially evanescent wave amplification, are of interest to us because the magnetic resonant coupling is essentially evanescent wave coupling. At resonance, EM fields are confined mostly inside the resonators, and electric energy and magnetic energy exchange periodically. Outside the resonators, the EM fields decay evanescently and do not carry away energy, unless coupled to the tail of the evanescent wave of another resonator. With a NIM, the amplitude of evanescent waves can be enhanced such that the virtual distance between two resonators are smaller. So the coupling coefficient of two resonators can be improved with a NIM slab.

\section{Transfer efficiency improvement}

The proposed idea is investigated with numerical simulations. Consider two identical dielectric resonators of infinite length, as shown in Fig. 1(a). Whispering-gallery modes exist in such resonators and can be excited by a line source inside the first resonator (the left resonator in Fig. 1(a)). Strong field is localized inside the first resonator and the field outside decays exponentially. Also we see that the same resonant mode is excited in the second resonator on right, with smaller field amplitude. In Fig. 1(b), two NIM slabs are inserted in the system, with $\varepsilon$ and $\mu$ both equal to -1 . The resonant mode is again excited by a line source in the left resonator; 
strong field is localized in the resonator and decays outside. However, when the field encounters with the NIM, the field is amplified and the evanescent field is extended further toward the second resonator. As a result, the coupling to the second resonator is stronger.

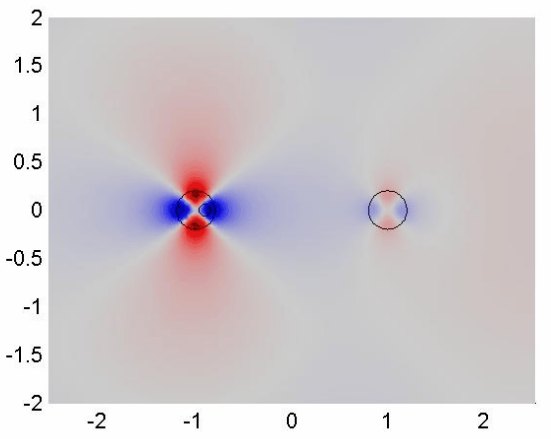

(a)

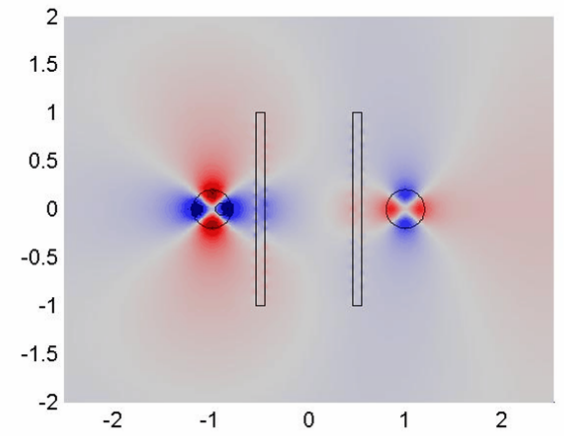

(b)

Figure 1 The coupling of two dielectric resonators (a) without and (b) with NIM slabs. The electric field distribution is shown in the figure.

A wireless power transfer system based on magnetic resonant coupling is also investigated. The system is based on coupled resonant coils, as shown in Fig. 2(a). The power is provided to a power loop, which feeds a source coil resonator inductively. The source coil is coupled resonantly to a sink resonator and a load loop is used to extract power from the sink resonator inductively. For a given distance between source and sink resonator $d$, the distance from power loop to source coil and the distance from sink coil to load loop $d_{l}$ need to be adjusted to optimize the coupling of the system in order to achieve highest efficiency. The parameters of the coil are designed to have a resonant frequency about $10 \mathrm{MHz}$. The system is modeled and calculated with COMSOL, which is a commercial simulation package based on finite-element method. The efficiency is calculated from the ratio of the transmitted power to the load loop to the input power of the power loop. For $d=1.8 \mathrm{~m}$, the efficiency of the system as a function of frequency is calculated and plotted in Fig. 2(b). A peak is observed at the resonance and the maximum efficiency is about $33 \%$.

For comparison, two NIM slabs are added to the system, with a thickness of $5 \mathrm{~cm}$ each. All other parameters are the same with the original system and the same procedure is applied to calculate the transfer efficiency of the new system. The peak efficiency of the new system is now over $50 \%$, which is a significant increase compared to the original system. Also because of the existence of the NIMs, the peak efficiency is shifted a little due to the coupling of NIMs with the resonators.

To apply metamaterials in real systems, the loss and dispersion of the metamaterials need to be accounted for. This need to be further studied in the future. Although the example shown here is for magnetic resonant coupled system, the proposed idea is a general approach for evanescent wave coupled systems, and can be used for electrically coupled systems too. 


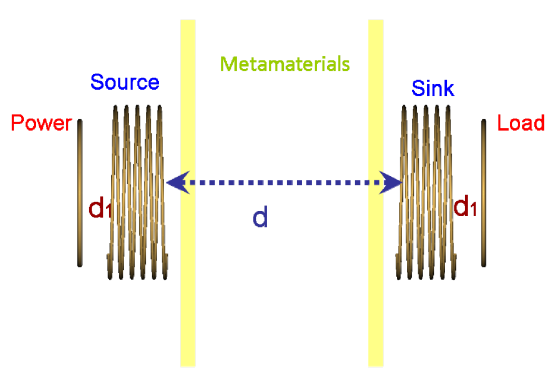

(a)

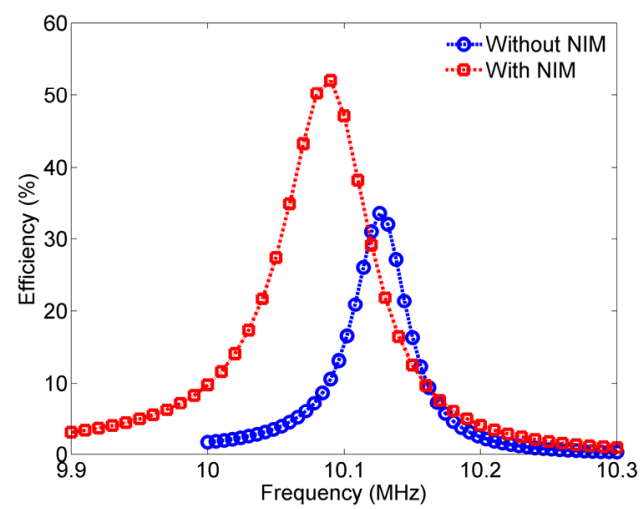

(b)

Figure 2 (a) An illustration of the wireless power transfer system model. The transmitter is composed of a power loop and a source coil resonator; the receiver is composed of a sink coil resonator and a load loop. For comparison, a new system is formed by adding NIM slabs to the system. (b) The calculated efficiency of system with and without the NIM slabs as a function of frequency.

\section{Conclusions}

In conclusion, the idea to use negative-index metamaterials to improve the efficiency of resonant energy transfer is proposed. We show with numerical studies that the coupling coefficient of two resonators can be enhanced by NIMs. The efficiency of a wireless power transfer system can also be improved with the help of metamaterials.

\section{References:}

[1] G. Vandevoorde and R. Puers, "Wireless energy transfer for stand-alone systems: a comparison between low and high energy applicability," Elsevier, Sensors and Actuators A: Physical, 92, 305 (2001)

[2] J. C. Schuder, H. E. Stephenson and J. F. Townsend, "High-level electromagnetic energy transfer through a closed chest wall," Inst. Radio Engrs. Int Conv. Record, 9, 119 (1961)

[3] N. de N. Donaldson and T. A. Perlins, "Analysis of resonant coupled coils in the design of radio frequency transcutaneous links," Med. \& Biol. Eng. \& Comput., 21, 612 (1983)

[4] R. Puers, K. V. Schuylenbergh, M. Catrysse and B. Hermans, "Wireless inductive transfer of power and data," Analog Circuit Design, p395 (2006)

[5] A. Karalis, J. D. Joannopoulos and M. Soljacic, "Efficient wireless non-radiative mid-range energy transfer," Annals of Physics, 323, 34 (2008)

[6] A. Kurs, A. Karalis, R. Moffatt, J. D. Joannopoulos, P. Fisher and M. Soljiacic, "Wireless power transfer via strongly coupled magnetic resonances," Science, 317, 83 (2007)

[7] D. R. Smith, J. B. Pendry, and M. C. K. Wiltshire, "Metamaterials and negative refractive index," Science, 305, 788 (2004)

[8] V. G. Veselago, "The electrodynamics of substances with simultaneously negative values of $\varepsilon$ and $\mu$," Sov. Phys. Usp., 10, 509 (1968)

[9] J. B. Pendry, "Negative refraction makes a perfect lens," Phys. Rev. Lett. 85, 3966 (2000)

[10] R. A. Shelby, D. R. Smith and S. Schultz, "Experimental Verification of a Negative Index of Refraction," Science, 292, 77 (2001) 